\title{
Antibiogram of Bacterial Isolates from Endotracheal Aspirates in a Tertiary Care Hospital in North India
}

\author{
Kanishtha Sharma, Suharshi Gupta*, Shashi Sharma and Perika Sharma \\ Department of Microbiology, Government Medical College, Rajouri, J\&K. India \\ *Corresponding author
}

\begin{abstract}
A B S T R A C T

\begin{tabular}{|l|}
\hline K e y w o r d s \\
Antibiotic policy, \\
Bacteriological, \\
Critically, \\
Education \\
programmes, Multi- \\
drug resistant \\
\hline Article Info \\
\hline $\begin{array}{l}\text { Accepted: } \\
\text { 20 May } 2019 \\
\text { Available Online: } \\
\text { 10 June 2019 }\end{array}$ \\
\hline
\end{tabular}

Respiratory tract infections are very common in critically ill patients in hospital settings. Endotracheal aspiration is a simple and easy method to perform especially in ICU patients for obtaining samples from lower respiratory tract. Therefore, this study was carried out to determine the microbiological spectrum and antibiotic susceptibility profile of Endotracheal aspirates among patients admitted in our institute. The present study was conducted on 348 ETT aspirate samples received in the Department of Microbiology, GMC, Jammu for a period of 1 year i.e. April 2017-2018. Bacteria were identified using standard bacteriological and biochemical methods. $285(81.89 \%)$ samples were culture positive. The most commonly isolated bacteria were Acinetobacter, followed by Klebsiella, Pseudomonas and Staphylococcus aureus. Meropenem, Amikacin and Tobramycin showed good sensitivity against GNB. Colistin, Polymyxin-B, Cefepimetazobactam were highly effective against Acinetobacter and Pseudomonas isolates. Multidrug resistant microorganisms are on a rise in our hospital. Strict implementation of antibiotic policy and creating awareness through education programmes can help in combating rapid spread of drug resistance.

\section{Introduction}

Respiratory infections in critically ill patients are associated with high morbidity and mortality. Investigation of the lower respiratory tract infections in hospitalized patients particularly in ICUs is difficult due to limited access to the site of infection. Endotracheal aspiration is a relatively noninvasive method that can be easily performed for obtaining early, accurate and representative sample of lower respiratory tract pathogens (Mouli et al., 2016). Once respiratory infection is diagnosed, treatment

strategy is early, swift administration of antibiotics as per culture results.

The etiological agents may vary according to duration of stay in hospital, the population of patients admitted, pre-existing illness and prior antimicrobial therapy. Internationally several studies done on ET aspirates reported predominantly gram negative organisms including Klebsiella spp, Pseudomonas spp, Enterobacter spp, Acinetobacter spp. and gram positive bacteria like Staphylococcus aureus. In India studies have been carried out to evaluate the organisms isolated from the
\end{abstract}


ET aspirates and demonstrated the significant isolation of gram negative organisms including Klebsiella spp, Acinetobacter spp, Pseudomonas spp. (A. Swati et al., 2018)

Appropriate antimicrobial stewardship that includes optimal selection, dose, and duration of treatment, as well as control of antimicrobial use, will prevent or slow the emergence of resistance among microorganisms in the hospital settings. (S. Priyanka et al., 2015) With this objective in mind this study was carried out to determine the microbiological spectrum and antibiotic susceptibility profile of Endotracheal aspirates among patients admitted in our institute. This will provide a valuable guidance for antibiotic therapy.

\section{Materials and Methods}

\section{Study design}

This was a retrospective study conducted in the Department of Microbiology, Government Medical College, Jammu. All endotracheal aspirate specimens received in the Microbiology department for culture and sensitivity testing over a period of 1 year i.e. from April 2017- April 2018 were included in the study.

\section{Sample collection}

\section{Endotracheal aspirate}

Sampling was done by introducing a catheter aseptically through the endotracheal tube and secretions aspirated into a sterile syringe.

\section{Endotracheal tube tip}

The endo-tracheal tube tip was cut aseptically into a sterile container and sent to the Microbiology laboratory.

\section{Sample processing}

Sample processing was done using standard aseptic precautions.

\section{Endotracheal aspirate}

$1 \mu \mathrm{l}$ of the specimen was inoculated on Blood agar and MacConkey agar.

\section{Endotracheal tube tip}

ET tip was rubbed on Blood agar and MacConkey agar plates for inoculation of sample.

\section{Bacterial isolation and identification}

A Gram stain of the endotracheal secretions/endotracheal tube tip fluid was done to assess the number of pus cells and the presence of bacteria. The inoculated plates were incubated overnight at $37^{\circ} \mathrm{C}$. All plates were examined for visible growth. The colonies were identified as per standard microbiological procedures. The bacteria were identified by their colony morphology, Gram staining, biochemical and sugar fermentation tests. Antibiotic sensitivity testing of the microorganisms was done by modified Kirby Bauer Disc Diffusion method on Muller Hinton agar and the results were interpreted as was recommended by the CLSI (Clinical Laboratory Standards Institute) guidelines.

\section{Results and Discussion}

ET tube tip provides the most representative sample of the pathogenic bacteria without any chance of contamination. Out of the 348 Endotracheal aspirates received in the Microbiology laboratory for analysis, 285 $(81.89 \%)$ were culture positive. 316 isolates were obtained from 285 culture positive samples i.e. $254(89.12 \%)$ yielded single 
bacterial isolate and $31(10.87 \%)$ yielded two bacterial isolates.

Among the isolates, Gram negative bacilli accounted $268(84.81 \%)$, Gram positive cocci $46(14.55 \%)$ and $2(0.63 \%)$ isolates of Candida species were obtained (Table 1).

Acinetobacter sp. was the predominant Gram negative bacilli which constituted 96 (35.82\%) followed by Klebsiella sp. 76 (28.35\%), Pseudomonas sp. 41 (15.29\%), Citrobacter sp. 24 (8.95\%), Escherichia coli 16 (5.97\%), Proteus sp. 10 (3.73\%), Enterobacter sp. 4(1.49\%) and Serretia $1(0.37 \%)$. Similar results were noted by (Modi et al., 2011) and (Mouli et al., 2016). Acinetobacter sp. is a persistent colonizer due to its ability of forming biofilm which is an important virulence factor for device associated infections (Ejaz et al., 2016)

Among the Gram positive bacteria Staphylococcus aureus 40 (86.95\%) was the leading isolate. Less frequently encountered organisms were Streptococcus pneumoniae and Enterococcus sp 3 (6.52\%) isolates each.

The culture positive specimens belonged to $188(65.96 \%)$ male and $97(34.03 \%)$ female hospitalized patients.

During the study it was noted that most of the isolates were obtained from intubated patients in MICU 129 (40.82\%) and EICU 65 $(20.56 \%$ ) (Table 2). This is a matter of concern as patients admitted in these units are seriously ill and are more prone to get infection. Our findings are in agreement with (Khosravi et al., 2013).

Antimicrobial resistance is becoming a critical issue in hospital settings, both for the management and prevention of serious infections in patients (Ejaz et al., 2016). It leads to increase in cost of patient care and also a rise in morbidity and mortality rate.
Studying the antibiogram of the bacterial isolates, both gram negative and gram positive bacteria showed multi-drug resistance (Table 3 and 4). Similar results were seen in many studies (Vedavathy et al., 2016). The major reasons for multidrug resistance are ventilators, prolonged hospital stay and irrational usage of antibiotics. (Santharam et al., 2018).

The non- fermenters Acinetobacter sp. and Pseudomonas sp. showed high sensitivity to Cefoperazone-sulbactam (88.54\%, 95.12\%), Colistin (87.5\%, 87.54\%), Polymyxin-B $(84.37 \%, \quad 92.68 \%)$, Cefepime-tazobactam $(92.70 \%, 90.24 \%)$ respectively. This is in concordance with Mst et al., (2018). Hence, its use should be restricted to severe nosocomial infections, in order to avoid rapid emergence of resistant strains.

Studying the antibiotic sensitivity profile of the Enterobacteriaceae most isolates showed high resistance pattern. The sensitivity pattern of Escherichia coli and Klebsiella sp. were as follows: Amoxycillin-clavulanic acid $(6.25 \%$, 1.31\%), Piperacillin-tazobactum (31.25\%, $50 \%)$ Ceftriaxone $(56.25 \%, 55.26 \%)$, Meropenem (87.5\%, 81.57\%), Amikacin (75\%, 68.42\%), Gentamicin (56.25\%, $55.26 \%)$, Tobramycin $(75 \%, 72.36 \%)$, Ciprofloxacin $(6.25 \%, \quad 7.89 \%)$, Cotrimaxazole $(12.5 \%, 6.57 \%)$ respectively. Similar result was seen in study conducted by Syal et al., (2018). Aminoglycosides and Quinolones are a good option for treatment but due to their unnecessary injurious use, resistance against them is also on a rise.

The antibiotic profile of Citrobacter $s p$. showed poor sensitivity to Cefepime (62.5\%), Cefuroxime (29.16\%), Ceftriaxone (58.33\%). High rate of resistance shows that Cephalosporins in monotherapy are no longer an effective treatment option. 
Int.J.Curr.Microbiol.App.Sci (2019) 8(6): 2562-2567

Table.1 Isolate frequency in endotracheal aspirate samples

\begin{tabular}{|c|c|c|c|c|c|c|}
\hline Total & Total & Single type & Two types & Bact & & Fungi \\
\hline 348 & 316 & 254 & 31 & $\begin{array}{c}\text { GNB } \\
268\end{array}$ & $\begin{array}{c}\text { GPC } \\
46\end{array}$ & 2 \\
\hline
\end{tabular}

Table.2 Ward-wise distribution of various isolates

\begin{tabular}{|c|c|c|c|c|c|c|c|c|c|c|c|c|c|}
\hline Wards & E.coli & Kle & Citro & Enb & Pseu & Acinet & Prot & Ser & Stap & Strep & Ent & BY & Total \\
\hline EICU & 8 & 17 & 4 & 3 & 10 & 18 & 2 & - & & 1 & 2 & - & 65 \\
\hline MICU & 4 & 36 & 10 & 1 & 6 & 51 & 4 & 1 & 12 & 2 & - & 2 & 129 \\
\hline SICU & 1 & 7 & 2 & - & 15 & 11 & - & - & 7 & - & - & - & 43 \\
\hline PICU & 2 & 12 & 4 & - & 5 & 13 & 3 & - & 6 & - & 1 & - & 46 \\
\hline $\begin{array}{c}\text { Surgery } \\
\text { ward }\end{array}$ & 1 & 4 & 4 & - & 5 & 3 & 1 & - & 7 & - & - & - & 25 \\
\hline
\end{tabular}

E.coli=Escherichia coli, Kle=Klebsiella, Citro=Citrobacter, Enb=Enterobacter, Pseu=Pseudomonas,

Acinet=Acinetobacter, Prot=Proteus, Ser=Serretia,, Stap=Staphyloccus, Strep=Streptococcus, Ent=Enterococcus,

$\mathrm{BY}=$ Budding yeast

Table.3 Antibiotic sensitivity profile of gram negative isolates $(\mathrm{N}=268)$

\begin{tabular}{|c|c|c|c|c|c|c|c|c|}
\hline & $\begin{array}{c}\text { E.coli } \\
(\mathrm{N}=16)\end{array}$ & $\begin{array}{c}\text { Kle } \\
(\mathbf{N}=76)\end{array}$ & $\begin{array}{c}\text { Citro } \\
(\mathrm{N}=24)\end{array}$ & $\begin{array}{c}\text { Enb } \\
(\mathrm{N}=4)\end{array}$ & $\begin{array}{c}\text { Pseu } \\
(\mathrm{N}=41)\end{array}$ & $\begin{array}{l}\text { Acinet } \\
(\mathrm{N}=96)\end{array}$ & $\begin{array}{c}\text { Prot } \\
(\mathrm{N}=10)\end{array}$ & $\begin{array}{c}\text { Ser } \\
(\mathbf{N}=1)\end{array}$ \\
\hline AMP & $0(0 \%)$ & $3(7.89 \%)$ & $2(8.33 \%)$ & $0(0 \%)$ & $5(12.19)$ & $10(10.41 \%)$ & $2(20 \%)$ & $0(0 \%)$ \\
\hline AMC & $1(6.25 \%)$ & $1(1.31 \%)$ & $0(0 \%)$ & $0(0 \%)$ & $0(0 \%)$ & $4(4.16 \%)$ & $1(10 \%)$ & $0(0 \%)$ \\
\hline PIT & $5(31.25 \%)$ & $38(50 \%)$ & $10(54.16 \%)$ & $2(50 \%)$ & $31(75.60 \%)$ & $65(67.70 \%)$ & $4(40 \%)$ & $1(100 \%)$ \\
\hline CPM & $8(50 \%)$ & $45(59.21 \%)$ & $15(62.5 \%)$ & $2(50 \%)$ & $33(80.48 \%)$ & $70(72.91 \%)$ & $5(50 \%)$ & $0(0 \%)$ \\
\hline CXM & $0(0 \%)$ & $6(7.89 \%)$ & $7(29.16 \%)$ & $1(25 \%)$ & $10(24.39 \%)$ & $20(20.83 \%)$ & $0(0 \%)$ & $0(0 \%)$ \\
\hline CTR & $9(56.25 \%)$ & $42(55.26 \%)$ & $14(58.33 \%)$ & $2(50 \%)$ & $21(51.21 \%)$ & $51(53.12 \%)$ & $2(20 \%)$ & $0(0 \%)$ \\
\hline AO & $12(75 \%)$ & $51(67.10 \%)$ & $16(66.66 \%)$ & $1(25 \%)$ & $30(73.17 \%)$ & $60(62.5 \%)$ & $2(20 \%)$ & $1(100 \%)$ \\
\hline MR & $14(87.5 \%)$ & $62(81.57 \%)$ & $21(87.5 \%)$ & $3(75 \%)$ & $35(85.36 \%)$ & $68(70.83 \%)$ & $8(80 \%)$ & $1(100 \%)$ \\
\hline AK & $12(75 \%)$ & $52(68.42 \%)$ & $18(75 \%)$ & $2(50 \%)$ & $32(78.04 \%)$ & $65(67.70 \%)$ & $6(60 \%)$ & $1(100 \%)$ \\
\hline GEN & $9(56.25 \%)$ & $42(55.26 \%)$ & $15(62.5 \%)$ & $3(75 \%)$ & $28(68.29 \%)$ & $58(60.41 \%)$ & $5(50 \%)$ & $1(100 \%)$ \\
\hline TOB & $12(75 \%)$ & $55(72.36 \%)$ & $20(83.33 \%)$ & $4(100 \%)$ & $35(85.36 \%)$ & $72(75 \%)$ & $6(60 \%)$ & $1(100 \%)$ \\
\hline CIP & $1(6.25 \%)$ & $6(7.89 \%)$ & $5(20.83 \%)$ & $1(25 \%)$ & $21(51.21 \%)$ & $48(50 \%)$ & $1(10 \%)$ & $0(0 \%)$ \\
\hline COT & $2(12.5 \%)$ & $5(6.57 \%)$ & $3(12.5 \%)$ & $1(25 \%)$ & $12(29.26 \%)$ & $20(20.83 \%)$ & $1(10 \%)$ & $0(0 \%)$ \\
\hline $\begin{array}{l}\text { CEF- } \\
\text { SUL }\end{array}$ & - & - & - & - & $39(95.12 \%)$ & $85(88.54 \%)$ & - & - \\
\hline CL & - & - & - & - & $36(87.80 \%)$ & $84(87.5 \%)$ & - & - \\
\hline PB & - & - & - & - & $38(92.68 \%)$ & $81(84.37 \%)$ & - & - \\
\hline $\begin{array}{l}\text { CPM- } \\
\text { TZ }\end{array}$ & - & - & - & - & $37(90.24 \%)$ & $89(92.70 \%)$ & - & - \\
\hline
\end{tabular}


Table.4 Antibiotic sensitivity profile of gram positive isolates $(\mathrm{N}=46)$

\begin{tabular}{|l|c|c|c|}
\hline & $\begin{array}{c}\text { Stap. } \\
(\mathbf{N = 4 0})\end{array}$ & $\begin{array}{c}\text { Strep. } \\
(\mathbf{N = 3})\end{array}$ & $\begin{array}{c}\text { Enterococcus } \\
(\mathbf{N}=3)\end{array}$ \\
\hline PG & $7(17.5 \%)$ & $1(33.3 \%)$ & $1(33.3 \%)$ \\
\hline VA & $36(90 \%)$ & $3(100 \%)$ & $2(66.6 \%)$ \\
\hline CD & $30(75 \%)$ & $3(100 \%)$ & $3(100 \%)$ \\
\hline E & $11(27.5 \%)$ & $2(66.6 \%)$ & $1(33.3 \%)$ \\
\hline LZ & $36(90 \%)$ & $3(100 \%)$ & $3(100 \%)$ \\
\hline C & $37(92.5 \%)$ & $2(66.6 \%)$ & $3(100 \%)$ \\
\hline GEN & $18(45 \%)$ & - & - \\
\hline CIP & $20(50 \%)$ & - & - \\
\hline COT & $23(57.5 \%)$ & - & - \\
\hline TET & $36(90 \%)$ & - & - \\
\hline CX & $25(62.5 \%)$ & - & - \\
\hline CEF & $10(25 \%)$ & - & - \\
\hline CTR & - & $2(66.6 \%)$ & $2(66.6 \%)$ \\
\hline CPM & - & $3(100 \%)$ & $2(66.6 \%)$ \\
\hline
\end{tabular}

Pencillin $\mathrm{G}=\mathrm{PG}, \mathrm{VA}=$ Vancomcin, $\mathrm{CD}=$ Clindamycin, $\mathrm{E}=$ Erythromycin, $\mathrm{LZ}=$ Linezolid, $\mathrm{C}=$ Chloramphenicol, GEN=Gentamycin, CIP=Ciprofloxacin, COT= Co-trimoxazole, TET $=$ Tetracycline, $\mathrm{CX}=$ Cefoxitin, $\mathrm{CEF}=$ Ceftazidime, $\mathrm{CTR}=$ Ceftriaxone, $\mathrm{CPM}=$ Cefipime

The Proteus sp. isolates showed high resistance against Ampicillin (20\%), Amoxycillin- clavulanic acid (10\%), Piperacillin-tazobactum (40\%), Ciprofloxacin $(10 \%)$ and Co-trimaxazole (10\%). Good sensitivity was seen against Meropenem $(80 \%)$.

Antibiotic sensitivity pattern of Gram positive isolates revealed good sensitivity of Staphylococcus aureus, Streptococcus sp. and Enterococcus sp. against Vancomycin, Linezolid and Chloramphenicol. 15 (37.5\%) isolates of Staphylococcus aureus were methicillin resistant. Similar results were seen in studies conducted by Chandra et al., (2017).

Our study concluded that multi drug resistant micro-organisms are on a rise in our hospital setting especially in ICU patients. Amongst Endotracheal tube aspirates the commonest organisms isolated were Acinetobacter, followed by Klebsiella and Pseudomonas.
There was an alarming increase of resistance against Penicillins, Cephalosporins and Quinolones. Imipenems still show good sensitivity but resistance is on a rise. Antibiotics such as Colistin and Polymyxin-B were very effective against Acinetobacter and Pseudomonas infections and should be used as reserve drugs. In order to fight the high antimicrobial resistance there is a need for development of strict antibiotic policy in the hospital so that injurious and irrational use of antibiotics is prevented. The infection control committee plays an important role in implementation of hand hygiene practices and creating awareness through education programmes.

\section{References}

Chandra, D., Avinash Laghawe, A., K. Sadawarte, K., Prabhu, T. 2017. Microbiological Profile and Antimicrobial Sensitivity Pattern of Endotracheal Tube Aspirates of Patients 
in ICU of a Tertiary Care Hospital in Bhopal. Int.J.Curr.Microbiol.App.Sci. 6(3): 891-895.

Ejaz, A., Tarar, M.R., Naeem, T., Naeem, M.A., Ijaz, S., Qureshi, M. 2016. Frequency of Multidrug resistant and extensively drug resistant organisms in tracheal aspirates. Biomedica. 32(2): 77-82.

Khosravi, A.D., Parhizgari, N., Abbasi, M. E., Mozaffari, A., Abbasi, F. 2013. The Prevalence of Bacteria Isolated From Endotracheal Tubes of Patients in Golestan Hospital, Ahvaz, Iran, and Determination of Their Antibiotic Susceptibility Patterns. Jundishapur J Microbiol. 6(1): 67-71.

Modi, P., Javadekar, T., Javadekar, B. 2011. Development of Bacteremia in ventilator associated pneumonia patients at a tertiary care hospital, Gujarat- A prospective study. National Journal of Medical Research. 1(1): 23 26.

Mouli, H.C., Nagaraja, M., Chandra, A., Kalawat, U. 2016. Endotracheal tube tip culture in post-operative respiratory infections in open heart surgery patients: a one year prospective study. J Clin Sci Res. 5(1): 214-20.

Mst, N.K., Shamsuzzaman, S.M., Fardows, J., Siddique, A.B., Joly, S.N. 2018. Identification of Bacterial Isolates from Endotracheal Aspirate of Patients in Intensive Care Unit and Their Antimicrobial Susceptibility Pattern.
8(2): 67-73.

Priyanka, S., Parihar, R. S., Khatri, P. K., Soni, L.K., A. Ramesh., D. Swati., and V. Shashikala. 2015. Antibiogram of bacterial isolates From, Tracheal aspirate of ICU patients at tertiary care hospital in western, Rajasthan, India. International Journal of Current Research. 7(7): 18125-18129.

Santharam, P., Sudha, K., Shanmugavadivoo, N., Usha, B., Padmavathi, B.K. 2018. Active Surveillance of endo-tracheal aspirates from mechanically ventilated. National Journal of Basic Medical Sciences. 8(3): 142-148.

Swati, A., Yamini, K., Rajkumar, R.V. 2018. Microbiological spectrum and antimicrobial susceptibility patterns of various isolates from endotracheal tube aspirates in a tertiary care hospital, Hyderabad, Telangana. Indian Journal of Microbiology Research. 5(2): 202207.

Syal, K., Singh, D., Thakur, A., Goyal, A., 2018. Micro-Organism Profile and Antibiotic Susceptibility Patterns in General ICU of Tertiary Care Hospital Situated in Hills. J Intensive Crit Care. 4(1):1-6.

Vedavathy, S., Sangamesh. 2016. Clinical study of ventilator associated pneumonia in a tertiary care centre. International Journal of Contemporary Pediatrics. 3(2): 432-41.

\section{How to cite this article:}

Kanishtha Sharma, Suharshi Gupta, Shashi Sharma and Perika Sharma. 2019. Antibiogram of Bacterial Isolates from Endotracheal Aspirates in a Tertiary Care Hospital in North India. Int.J.Curr.Microbiol.App.Sci. 8(06): 2562-2567. doi: https://doi.org/10.20546/ijcmas.2019.806.308 